\title{
Fetal Pathology Findings Domain
}

National Cancer Institute

\section{Source}

National Cancer Institute. Fetal Pathology Findings Domain. NCI Thesaurus. Code C95091.

A subject domain utilized for the submission of information encompassing and representing data, vocabulary or records related to fetal and neonatal developmental morphology. 